\title{
L-BAND ACTIVE / PASSIVE TIME SERIES MEASUREMENTS OVER A GROWING SEASON USING THE COMRAD GROUND-BASED SMAP SIMULATOR
}

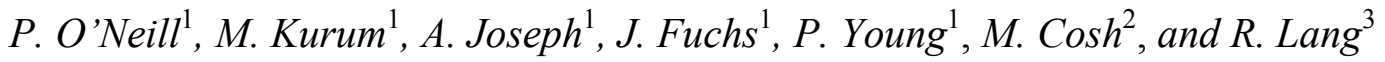 \\ ${ }^{1}$ Hydrological Sciences Laboratory / Code 617 \\ NASA Goddard Space Flight Center, Greenbelt, MD 20771 USA \\ tel: 1-301-614-5773, fax: 1-301-614-5808, Peggy.E.ONeill@nasa.gov \\ ${ }^{2}$ Hydrology and Remote Sensing Laboratory, U.S. Dept. of Agriculture/Agricultural Research Service ${ }^{*}$ \\ Beltsville, MD 20105 USA \\ ${ }^{3}$ Dept. of Electrical \& Computer Engineering, George Washington University \\ Washington, DC 20052 USA
}

\begin{abstract}
Once launched in late 2014, NASA's Soil Moisture Active Passive (SMAP) mission will use a combination of a four-channel L-band radiometer and a threechannel L-band radar to provide high resolution global mapping of soil moisture and landscape freeze/thaw state every 2-3 days. These measurements are valuable to improved understanding of the Earth's water, energy, and carbon cycles, and to many applications of societal benefit. In order for soil moisture to be retrieved accurately from SMAP microwave data, prelaunch activities are concentrating on developing improved geophysical retrieval algorithms for each of the SMAP baseline products. The ComRAD truckbased SMAP simulator collected active/passive microwave time series data at the SMAP incident angle of $40^{\circ}$ over corn and soybeans during 2012 for use in refining SMAP retrieval algorithms.
\end{abstract}

Keywords (Index Terms) -- soil moisture, microwave, geophysical retrievals, ComRAD, SMAP, corn, soybeans.

\section{INTRODUCTION}

Scheduled to launch in October, 2014, NASA's Soil Moisture Active Passive (SMAP) mission will provide high-resolution global mapping of soil moisture and freeze/thaw state every 2-3 days. These new measurements of the hydrological condition of the Earth's surface will build on data from ESA's current SMOS satellite to enable new science and applications of societal benefit that range from agriculture to human health. The SMAP instrument design incorporates an L-band radar (3 $\mathrm{km}$ resolution) and an $\mathrm{L}$ band radiometer (40 $\mathrm{km}$ resolution) sharing a single 6-meter rotating mesh antenna to produce an intermediate resolution $(9 \mathrm{~km})$ soil moisture. In the SMAP prelaunch time frame, algorithms are being developed for each of the SMAP baseline data products derived from these two instruments [1]. Refinement of these algorithms require coincident active/passive data sets at the SMAP frequency and incidence angle in time series over a long enough time period to reflect changing surface conditions (vegetation, soil moisture, etc.). As part of SMAP algorithm development activities, NASA GSFC's ComRAD truck-mounted microwave instrument system collected L-band active/passive data throughout the growing season over corn and soybean crops from June 1 through October 24, 2012, along with associated ground truth and vegetation characterization data. The resulting time series data will be used in refining the parameterizations in the SMAP baseline algorithms.

\footnotetext{
*USDA is an equal opportunity provider and employer.
} 


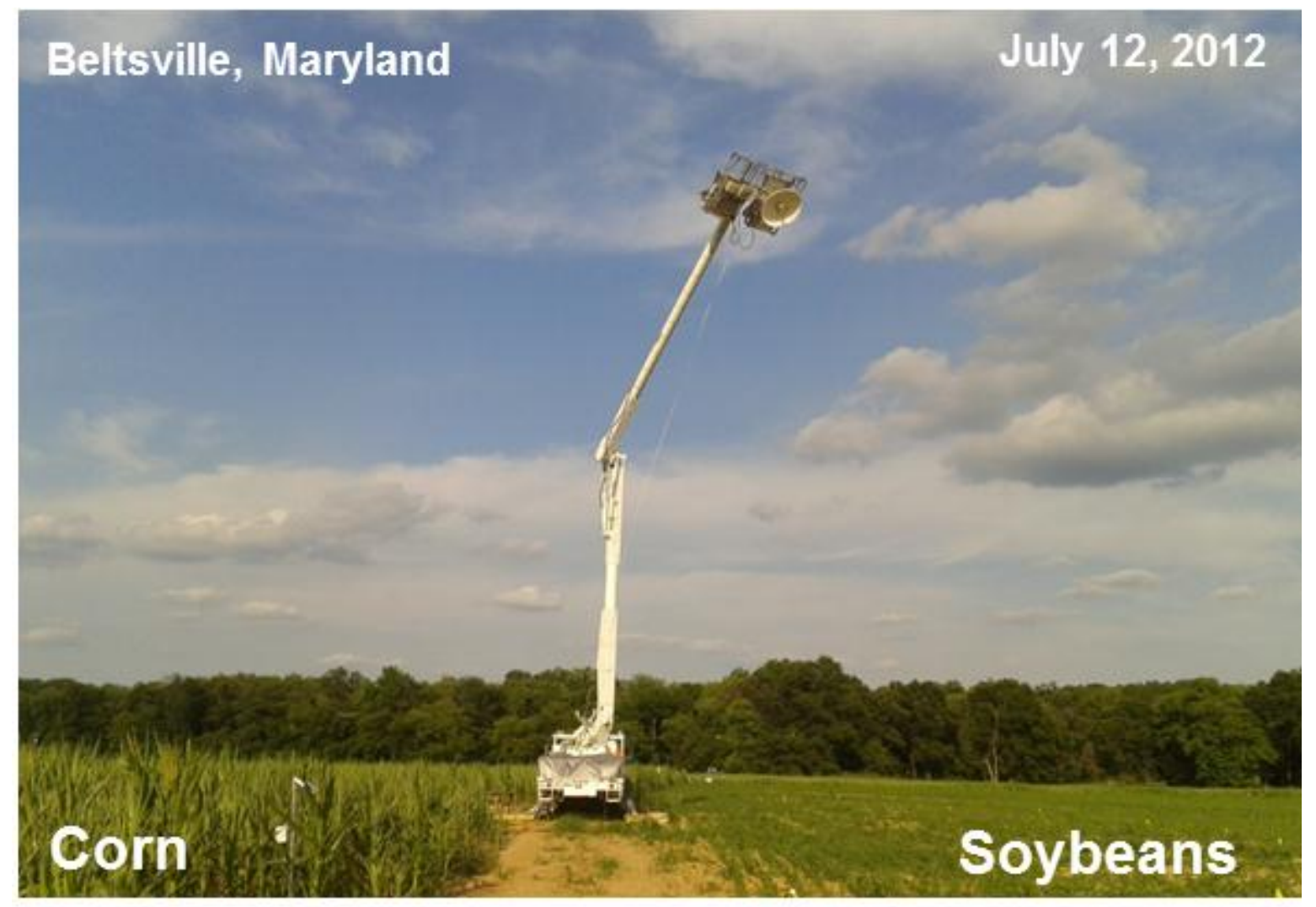

(a)

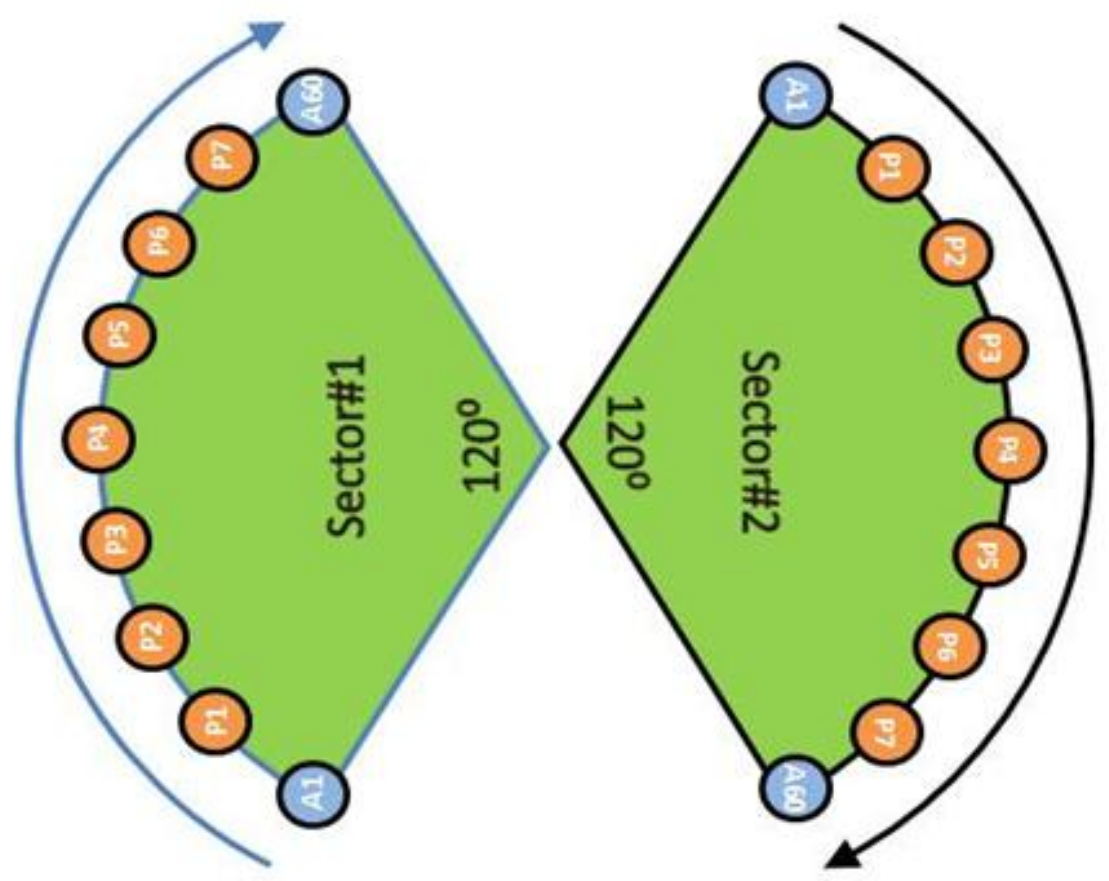

(b)

Figure 1. (a) ComRAD truck-mounted instrument system deployed at the USDA OPE3 test site during July, 2012. (b) Schematic of ComRAD data-taking positions over soybeans (sector \#2) and corn (sector \#1). Approximately 60 independent radar measurements were acquired during $120^{\circ}$ azimuthal sweeps of the boom over each crop (blue A1 - A60), while passive data were collected at 7 discrete locations (orange $\mathrm{P} 1$ through $\mathrm{P} 7$ ) within the $120^{\circ}$ sectors for each crop. 


\section{COMRAD INSTRUMENT SYSTEM}

The ComRAD (for Combined Radar / Radiometer system) microwave instrument system used in this investigation has been developed jointly by NASA/GSFC and George Washington University [2]. ComRAD includes a dual-pol 1.4 $\mathrm{GHz}$ radiometer and a quad-pol $1.24-1.34 \mathrm{GHz}$ radar sharing a new $1.22-\mathrm{m}$ Cassegrain parabolic dish antenna and subreflector [3] to achieve a very low loss system. Absolute accuracy and the sensitivity of the instrument are $\pm 1 \mathrm{~K}$ and $\pm 0.1 \mathrm{~K}$, respectively. External calibration is achieved using cold sky and ambient microwave absorber targets for the radiometer, and flat plates and dihedral reflectors for the radar. When deployed in the field, ComRAD is mounted on a $19-\mathrm{m}$ hydraulic boom truck (Fig. 1a) and can operate over a range of incidence angles from $0^{\circ}$ to $175^{\circ}$ and a $300^{\circ}$ range in azimuth. The mounting platform can also accommodate additional small instruments such as a CropScan visible/infrared sensor for vegetation reflectance measurements and a thermal infrared sensor for scene physical temperature.

\section{2012 FIELD EXPERIMENT}

An extensive field experiment from crop planting through senescence was conducted from June to October, 2012 at the heavily instrumented USDA-ARS (U.S. Dept. of AgricultureAgricultural Research Service) OPE3 (Optimizing Production Inputs for Economic and Environmental Enhancement) [4] test site in Beltsville, MD to acquire data needed to address active/passive microwave algorithm needs for accurate soil moisture retrieval. Vegetation cover in the experiment consisted of two crops, corn and soybeans, planted on either side of the ComRAD truck staging area (Figure 1a). Corn was planted at the site on May 16 and harvested on October 17-18; soybeans were planted approximately one month later on June 14 and harvested on October 26. In situ soil moisture, soil temperature, and leaf wetness sensors were installed by USDA to provide continuous ground truth data. These data were supplemented by additional soil moisture data collected manually twice a week by USDA personnel, along with weekly plant architectural, water content, and density measurements. The OPE3 site also contains a SCAN meteorological station and a flux tower which record precipitation and other micrometeorological data. ComRAD microwave measurements at the SMAP incidence angle of $40^{\circ}$ were made on 75 days between June 1 and October 24, 2012. Active and passive data were acquired autonomously every 90 minutes (weather permitting) accordingly to the schematic in Figure $1 \mathrm{~b}$. Manual calibration of the radiometer was performed weekly.

\section{ANALYSIS}

Time series data from the 2012 ComRAD field experiment will be used to refine soil moisture retrieval algorithms being developed for the SMAP mission. An example of a ComRAD time series over corn from a 5-day period in late summer is shown in Figure 2. Diurnal patterns as well as an overall drying trend can be seen in the plotted data. Similar data throughout the growing season should prove useful to improving the parameterizations (related to both changing vegetation conditions and to simultaneous active/passive responses) in many of the SMAP baseline algorithms.

\section{REFERENCES}

[1] Entekhabi, D., E, Njoku, P. O’Neill, K. Kellogg, plus 19 others, "The Soil Moisture Active Passive (SMAP) Mission," Proceedings of the IEEE, Vol. 98, No. 5, May, 2010.

[2] O’Neill, P.E., R.H. Lang, K.R. Carver, M. Kurum, and C. Utku, "Multi-Sensor Microwave Remote Sensing using NASA's Combined Radar/Radiometer (ComRAD) System," Proc. of MicroRad'06: Specialist Meeting on Microwave Radiometry and Remote Sensing Applications, San Juan, Puerto Rico, Feb. 28-March 3, 2006.

[3] Ogut, M., R. Lang, W. Wasylkiwskyj, M. Kurum, and P. O'Neill, "Performance of an L Band Antenna for Radiometric Measurements," 2013 National Radio Science Meeting, USNC_URSI, Boulder, CO, January 9-12, 2013.

[4] USDA web site at http://www.ars.usda.gov/Research/. 



Figure 2. Example of ComRAD time series microwave measurements over corn during August 27 September 1, 2012 at the SMAP incidence angle of $40^{\circ}$. [top] $L$ band brightness temperatures at horizontal (red) and vertical (blue) polarizations along with the scene infrared temperature (green). [bottom] $\mathrm{L}$ band radar backscatter at $\mathrm{HH}, \mathrm{VV}, \mathrm{HV}$, and $\mathrm{VH}$ polarizations acquired over the same time period as the passive data. 